\title{
Nutrients and Mother's Diet in the Epigenetic Regulation of Placenta
}

\section{Los Nutrientes y la Dieta de la Madre en la Regulación Epigenética de la Placenta}

\author{
Iván Enrique Naranjo Logroño ${ }^{1,2}$, Anthony Alfonso Naranjo Coronel ${ }^{2}$, Cristian \\ Alberto Zumárraga Pozo ${ }^{1}$, and María Belén Peñaherrera Lema ${ }^{1}$ \\ ${ }^{1}$ Carrera de Medicina, Facultad de Salud Pública, ESPOCH, Riobamba, Chimborazo, Ecuador \\ ${ }^{2}$ COLPOMED Centro, Hospital del Día, Riobamba, Chimborazo, Ecuador
}

XVIII International Seminar on Health, Food and Human Nutrition

Corresponding Author:

Iván Enrique Naranjo Logroño naranjometropolitana@hotmail.com

Published: 9 September 2021

Production and Hosting by Knowledge E

(c) Iván Enrique Naranjo Logroño et al. This article is distributed under the terms of the Creative Commons Attribution License, which permits unrestricted use and redistribution provided that the original author and source are credited.

\section{Abstract}

Introduction: Pregnancy was defined as a state that exposes women to multiple anatomicalphysiological, biochemical, psychological, and adaptive genomic changes in an environment of homeostatic balance and preparation of the fetus in the external environment. From fertilization to birth, a genetic program previously imprinted in your cells had been developed, which was influenced by the state of maternal health, preconception, nutritional factors, and the diet of the pregnant woman. Other secondary ones were of the maternal-fetal hormonal type, stress, toxic, viral or bacterial infections. Objective: To describe the influence of nutrition and maternal diet as factors of change in the epigenetic regulation of the placenta and in the perinatal results. Methods: Academic Google, Scopus, PubMed, ClinicalKey databases were searched using MeSH and DeCS terms: 'epigenetics', 'placent', 'diet', 'pregnancy', 'imprint'. Discussion: Methylation of deoxyribonucleic acid (DNA) was the common way for epigenetic expression to take place and could be demonstrated in tissues. Observations highlight differences in placental tissue and the existence of hypo-methylation zones of DNA that resemble those present in malignant cells, the placenta being very sensitive to epigenetic marks and to maternal and fetal signals that allow its adaptation or depress influences from the environment. Conclusion: The placenta had unique genomic characteristics and was a reactive tissue to internal and external influences that had great clinical importance for fetal growth, appearance of neural tube defects, development of diabetes, high blood pressure, obesity, and other extrauterine diseases.

Keywords: epigenetic, nutrition, placenta, methylation.

\section{Resumen}

Introducción: El embarazo es un estado que expone a la mujer a múltiples cambios anátomo-fisiológicos, bioquímicos, psicológicos, genómicos adaptativos en un entorno de equilibrio homeostático y de preparación del feto al medio externo. Desde la fecundación hasta el nacimiento se desarrolla una programación genética previamente impresa en sus células que se ven influenciadas por el estado de salud materna, factores preconcepcionales, nutricionales y la dieta de la gestante. Otros secundarios son del tipo hormonal maternofetales, el estrés, tóxicos, infecciones víricas o bacterianas. Objetivo: Describir la influencia de la nutrición y la dieta materna como factores de cambio en la regulación epigenética de la placenta y en los resultados perinatales. Métodos: Se realizaron búsquedas en bases de datos Academic Google, Scopus, PubMed, ClinicalKey utilizando términos MeSH y DeCS: 
'epigenética’, ‘placenta', 'dieta', ‘embarazo', ‘impronta’. Discusión: La metilación del Acido desoxirribonucleico (ADN) es la manera común para que la expresión epigenética tenga lugar y sea demostrada en los tejidos. Observaciones destacan diferencias en el tejido placentario y la existencia de zonas hipo metilación del ADN que se asemejan a las presentes en células malignas, siendo la placenta muy sensible a marcas epigenéticas y a las señales maternas y fetales que permiten su adaptación o deprimen las influencias del entorno. Conclusión: La placenta presenta características genómicas únicas y es un tejido reactivo a influencias internas y externas que le confiere gran importancia clínica para el crecimiento fetal, aparición de defectos del tubo neural, desarrollo de diabetes, hipertensión arterial, obesidad y otras enfermedades extrauterinas.

Palabras Clave: epigenética, nutrición, placenta, metilación.

\section{Introducción}

El concepto de epigenética y de programación prenatal se describe en un inicio de la medicina humana y posteriormente incluso en la veterinaria. Desde sus inicios se descubrió que la epigenética es la piedra angular del desarrollo de características como el crecimiento, aparición de obesidad, enfermedades no transmisibles como diabetes, trastornos renales y cardiovasculares, las cuales se van expresando o no desde la vida intrauterina. Todo lo concerniente a la epigenética relacionada con el embarazo se desarrolla a partir de estudios epidemiológicos, donde se logra descubrir que los niños con bajo peso al nacer a causa de afectaciones del crecimiento y del desarrollo fetal, presentaban una incidencia elevada de enfermedades tanto metabólicas como cardiovasculares cuando llegaban a ser adultos [1].

Posterior a los estudios encaminados a observar la asociación genómica, el análisis de rasgos y el mapeo epigenómico se estableció que se necesitan más esfuerzos para definir de manera acertada las interacciones genético-epigenéticas y comprender su papel en la susceptibilidad y progresión de alguna anomalía o enfermedad. Consecuentemente, el análisis de los efectos de las variaciones en la expresión génica y metilación del ácido desoxirribonucleico (ADN) en placentas humanas se considera que puede tener múltiples implicaciones mecanicistas y prácticas para el tratamiento y prevención de múltiples enfermedades relacionadas con el desarrollo gestacional, placentario y fetal [2].

c Los problemas que pudieran relacionarse con estos parámetros respecto a anomalías del crecimiento, desarrollo y maduración tisular; constituyen un capítulo importante para múltiples especialidades que deben mantener un seguimiento cercano del desarrollo fetal y del estado de nutrición materno. El fenómeno biológico del crecimiento está dado básicamente por hiperplasia y por consiguiente al aumento de masa corporal, en cambio, el desarrollo se relaciona con el grado de organización y complejidad a nivel estructural y funcional de las estructuras orgánicas [3, 4]. Finalmente, otro término utilizado es la maduración que se refiere al desarrollo y especialización de un tejido u organismo. Por tanto, durante el embarazo y dada su complejidad asociada 
al desarrollo de nuevos tejidos, órganos, aparatos y sistemas; deben confluir los tres procesos mencionados de manera armónica para que el feto pueda lograr adquirir capacidades necesarias para su supervivencia en el exterior $[3,5,6]$.

Por otra parte, desde que se produce la fecundación hasta que ocurre el nacimiento, existe la ejecución de la programación genética impresa en sus genes y puede ser modificada la expresión por el estado de la madre en la pregestación como durante la gestación, principalmente la nutrición y la influencia de la dieta pudiese marcar diferencias en el resultado; también se debe considerar los cambios e influencias hormonales maternas y fetales, estrés, factores tóxico-ambientales, infecciones, entre otros factores [3, 7-10]. Las primeras dos semanas del desarrollo embrionario son de suma importancia debido a que el embrión es altamente vulnerable, no solo a anomalías del desarrollo si no incluso es susceptible a la muerte antenatal [3, 6, 10-12].

Según refiere Herrera et al. Describe que el embarazo tiene dos fases una anabólica dedicada a elaborar y almacenar sustratos necesarios para la segunda etapa. Otra etapa es catabólica donde todas las reservas son utilizadas tanto para proporcionar material energético y estructural [5, 13].

El conocimiento de patologías hereditarias o existentes por modificaciones cromosómicas son parte central de la investigación perinatal y valoración de la salud neonatal. Las hipótesis de Barker o Palinski-Napoli, lograron el desarrollo de una multitud de estudios que valoran la interacción madre e hijo en sus fases del desarrollo, así como la necesidad de conocer los factores que regulan dicho desarrollo $[3,6,10,11]$.

Es conveniente para todas las especialidades conocimiento de los factores que pueden intervenir con un proceso materno-fetal normal, por ello la meta de este documento es describir la influencia de la nutrición y la dieta materna como factores de cambio en la regulación epigenética de la placenta y en los resultados perinatales.

\section{Método}

Se realiza un trabajo de revisión de artículos científicos en la literatura y cuyos criterios de inclusión toman en cuenta las investigaciones que centran su atención en temas relacionados con la influencia de la dieta y la epigenética en el embarazo, para lo cual se seleccionaron 30 estudios publicados desde el año 2013 hasta la actualidad y en la que se reconocen sólo artículos originales. Los criterios de exclusión principalmente fueron relacionados con artículos con temáticas referentes a epigenética fuera del embarazo, epigenética no relacionado con la dieta, artículos sin texto disponible e investigaciones que no contenían resultados explícitos, siendo eliminados 14 de un total de 44

publicaciones. Dentro de los artículos preseleccionados, se realizó un proceso de cribado y se tomó en cuenta sólo los artículos que contenían la relación de la dieta con la epigenética en mujeres embarazadas. Se optó por un proceso de búsqueda en bases de datos reconocidas, como: Academic Google, Scopus, PubMed, Lilacs, ClinicalKey, utilizando términos MeSH y DeCS y palabras claves como epigenética, nutrición, embarazo como descriptores principales. Todas las publicaciones deben cumplir con los criterios de inclusión y exclusión establecidos. 


\section{Resultados}

Los resultados de la búsqueda se organizaron de acuerdo con el interés y sentido del trabajo, y criterios de inclusión, luego de una discriminación de artículos, revisiones sistemáticas, ensayos de laboratorio relacionados con la búsqueda y no más de 5 años de antigüedad en las bases de datos, se logró obtener 30 artículos, que se sometieron a revisión para ser considerados y mencionados en él trabajo. Los artículos excluidos contenían información sin relevancia o evidencia sustentada, también trabajos publicados fuera de rango de tiempo permitido y sin actualizaciones en el tema. Se filtran e incluyen estudios que presentan interés para la discusión del tema.

\section{Discusión}

El embarazo es una etapa de mucha importancia para una mujer durante su ciclo vital y es en este periodo donde tienen lugar muchos cambios y procesos a nivel anatómico, fisiológico, emocional, genómico, entre muchos otros; que facilitan la adaptación de la madre a los cambios y al nuevo en desarrollo, además que prepara todo para la posterior adaptación al medio externo del feto y llevar a cabo el parto.

La importancia de saber que factores intervienen en el desarrollo de caracteres que pueden intervenir en la aparición y desarrollo de enfermedades o anomalías es que se puede intervenir y evitar dichas fallas para proporcionar una mejor calidad de vida tanto a la madre como al feto. Las primeras dos semanas del desarrollo embrionario son de suma importancia debido a que el embrión es altamente vulnerable, no solo a anomalías del desarrollo si no incluo es susceptible a la muerte antenatal. El periodo entre la segunda y octava semana el embrión es extremadamente sensible a agentes teratógenos, puesto que este tiempo coincide con la formación de los primordios de órganos importantes y es en este punto, donde pueden aparecer graves malformaciones las cuales pueden ser alteraciones leves o algunas ser de gran importancia y producir dificultades para las adaptaciones fisiológicas [3, 6, 10-12].

Según refiere Herrera et al. El embarazo, acontece dos etapas, la primera o anabólica, es un proceso de elaboración de reservas que serán utilizadas en la siguiente etapa. La segunda etapa o catabólica tiene lugar la movilización de reservas para el desarrollo fetal y la adaptación externa tras el parto. El periodo anabólico corresponde a picos de insulina y aumento a la sensibilidad en el organismo materno; también se observa el crecimiento placentario, aumento del volumen amniótico, incremento de las 'reservas proteicas y lipídicas' en la madre. En la fase catabólica se produce la distribución de reservas elaboradas y colaboran con el crecimiento placentario y aumento del líquido amniótico; se da un crecimiento fetal exponencial por lo que en consecuencia se produce una reducción del ritmo de ganancia de las reservas maternas puesto que todas estas contribuyen al desarrollo fetal $[5,13]$. 


\subsection{Teoría de Barker}

Conocida también como teoría de programación o el efecto programador intrauterino, donde se postula que existe una relación importante entre el desarrollo intrauterino y entorno. Se considera que si la madre se encuentra en un estado de mal nutrición se produce una agresión en el feto, subsecuentemente una mal nutrición fetal y anomalías en el desarrollo como el bajo peso al nacer (BPN) y el riesgo elevado de aparecimiento de una enfermedad cerebro vascular (ECV), también se evaluó varias hipótesis de los orígenes a nivel fetal de enfermedades coronarias, diabetes, hipertensión arterial, entre otras $[4-6,12,13]$.

\subsection{Características únicas de la placenta en epigenética}

Los estudios demuestran que la placenta a diferencia de los demás tejidos del cuerpo presenta características epigenéticas únicas, pero con mecanismos o funcionalidad similar $[14,15]$.

Las observaciones actuales destacan diferencias de la placenta en el ámbito epigenético, la principal es la existencia de zonas de menor nivel de metilación del ADN (ADN-M). Gracias a la investigación realizada por Schroeder et al. En 2013, se conoce que la placenta al igual que otros tejidos presenta células con altos niveles de metilación (>70\%), representando a la mayoría del genoma de este tejido, pero en alrededor del $37 \%$ del genoma placentario contiene zonas con menor nivel de metilación, recibiendo el nombre de Dominios Parcialmente Metilados (DPM), se le denomina de esta manera por la existencia pobre de genes, que a pesar de ser muy reactivos a la metilación, al ser escasos no se reflejan como espacios con alto nivel de ADN-M [16, 17]

Según, De Goede et al. En su revisión menciona que, por los pobres avances en el ámbito epigenético relacionado con el tejido placentario, aún es desconocida la función específica de los DPM, sin embargo, su presencia sugiere que existe alta organización y especialización del genoma placentario a diferencia de otras células [18].

Otra característica importante es la presencia de niveles variables de metilación de varias familias de elementos retro transponibles, teniendo en cuenta que en su mayoría se evidencia hipometilación [14]. Un elemento retrotransponible o retrotransposón se define como segmentos de ADN que tiene la capacidad de movimiento y replicación, antiguamente conocido como segmentos 'saltarines' que cambian de posición en las cadenas de ADN por lo que los retrotransposones son responsables de crear variaciones genéticas $y$, en ocasiones, mutaciones que causan enfermedades, dentro del genoma humano [19]. Por dicha razón, Reiss destaca en su trabajo investigativo que los retrotransposones están silenciados por una fuerte metilación del dinucleótido Citocina-Guanina ( $\mathrm{CpG}$ ) en genomas de mamíferos con el fin de evitar expresión de genes contraproducentes para el desarrollo embrionario y fetal [20].

Otra característica destacable de la placenta que la diferencia de los demás tejidos es su carácter de pseudomalignidad, observaciones comparativas del tejido placentario versus tejidos malignos han arrojado resultados sorprendentes, pues las características únicas de la placenta en relación con la metilación del ADN como la hipometilación y 
los DPM también se evidencian también en las células malignas, lo que sugieren que los dos tipos de tejidos tienen mecanismos epigenéticos comunes [21].

Semejante a los tejidos malignos, la placenta tiene propiedades altamente invasivas, prolifera en condiciones hipóxicas, evade las líneas de defensa huésped y logra obtener un suministro de sangre propio. Análogamente, en la placenta existe una tendencia hacia la metilación de genes supresores de tumores y la activación de oncogenes [14].

Finalmente cabe mencionar que la placenta tiene la propiedad de ser muy sensible a las marcas epigenéticas, gracias a esto, la placenta responde mejor a las señales que provienen de la madre y del feto, permitiendo una adaptación dependiendo de las necesidades y que amortigüe las influencias del entorno al que el feto puede estar expuesto [14]

\subsection{Marcas epigenéticas e impronta genética}

La metilación se da en sitios específicos del ADN para controlar la transcripción de genes, este proceso tiene ciertas peculiaridades que modifican la metilación y desmetilación dependiendo de algunas características como el desarrollo embrionario y el sexo del nuevo ser $[4,7,11,22,23]$.

La metilación del ADN ha sido desde el inicio la modificación epigenética más estudiada y está asociada al silenciamiento o inhibición génica cuando ocurre en la región promotora de los genes [14, 24].

Se ha observado que la metilación generalmente ocurre a nivel de los dinucleótidos CpG y no es más que la adición de un grupo metilo al quinto carbono de una citosina, originando una 5- metilcitosina (5-mC), todo este proceso se da mediante la acción de enzimas catalizadoras, a las cuales se las ha denominado ADN metiltransferasas (ADNMT) [14, 24, 25]. Profundizando un poco más acerca de las ADNMT, existen subtipos, denominados ADNMT1, ADNMT3A, ADNMT3B, recientemente se descubrieron otras enzimas que se cree oxidan la 5-mC, provocando la desmetilación del ADN, los subtipos reconocidos son TET1, TET2, TET3 [24-26].

Las marcas epigenéticas intervienen de manera importante en la regulación de la impresión genómica, este fenómeno epigenético es de vital importancia para el correcto desarrollo, maduración y función de la placenta, además participan en gran medida en el crecimiento fetal. Recientemente se ha vinculado las enzimas del ADN materno mitocondrial (ADNMTs) con la diferenciación y regulación de los trofoblastos placentarios [26].

La existencia de errores en la impresión de los genes puede ser causados por interrupciones epigenéticas que pueden dar como producto la presencia de diferentes fenotipos y dar paso a entidades nosológicas como la restricción del crecimiento fetal, abortos espontáneos, maduración acelerada de la placenta, nacimientos prematuros e incluso se vincula con la presentación de preeclampsia [14, 15, 24, 27].

Cabe mencionar que todo el proceso de metilación del ADN está regulado por Regiones de Control de Impresión, que de acuerdo con el estado de metilación del 
ADN tienen la capacidad de intervenir en la expresión de diversos genes impresos, este proceso generalmente ocurre en grupos o segmentos de material genético [24].

Conforme el embrión se desarrolla la metilación desaparece del genoma y posteriormente dependerá del sexo del embrión y restaurará su propio patrón, a esto se le llama impronta $[4,7,11,22,23]$.

\subsection{Programación fetal}

El establecimiento de las marcas epigenéticas en el desarrollo fetal está influenciado por factores ambientales, uno de los más importantes es la dieta [6].

Se dan dos etapas de desmetilación de secuencias codificantes (genes) y secuencias repetitivas (transposones) luego de la fertilización, sin embargo, en ambos casos, la metilación ocurre nuevamente de forma rápida. Hay genes que escapan a la metilación 'de novo', o sufren cambios en el patrón [8-10].

Muchos estudios han demostrado en los últimos años que enfermedades prevalentes como la diabetes, obesidad, hipertensión arterial y enfermedades cardiovasculares pueden tener su origen en la alimentación materna, gestación, lactancia, y pueden estar relacionadas con la metilación del ADN [6, 8-10].

\subsection{Ganancia peso}

En la gestación la madre debe conseguir una ganancia de peso entre 11,3 y 15,8 $\mathrm{kg}$ para asegurar que el nuevo ser tenga también un peso adecuado al nacimiento (entre 2,5 y $4,0 \mathrm{~kg})[4,6,13]$. Sin embargo, actualmente y considerando que tanto la gestación de productos Pequeños para la Edad Gestacional (PEG) como la de Grandes para la Edad Gestacional (GEG) presenta problemas graves y tienen gran riesgo de desarrollar a futuro enfermedades; además la ganancia excesiva o reducida de peso implica riegos para la madre, lo más recomendable es un incremento de peso dentro de parámetros normales durante el embarazo desde la fecundación hasta el nacimiento (peso pregestacional) [4, 11, 13].

Al finalizar la gestación aparecen 'ajustes' que se correlacionan con la transición desde el vientre a la lactancia [28]. Los ajustes se relacionan con cambios en la concentración de glucosa, lípidos, proteínas, hormonas como la insulina, progesterona, prolactina, somatotropina coriónica en la madre, teniendo en cuenta que la madre puede desarrollar resistencia a la insulina. Esto se da debido al mecanismo protector corporal materno para asegurar glucosa para el cerebro materno y fetal; además de dirigir glucosa a la glándula mamaria para la correcta formación de leche materna reduciendo la utilización de glucosa por otros tejidos de la madre [13].

\subsection{Diabetes gestacional (DG)}

En algunas mujeres sanas (no diabéticas), durante el embarazo se desarrolla una alteración en el metabolismo de los carbohidratos, a pesar de que en ayuno los 
niveles de glucosa e insulina suelen tener parámetros normales e incluso reducidos; tras el consumo de carbohidratos, el nivel de glucosa en sangre se eleva por sobre los valores normales. Estos cambios son más notorios en el último trimestre de la gestación $[3,12,13,23]$.

La DG ha sido estudiada de manera amplia en roedores, con resultados que se pueden asemejar a los del ser humano. Las ratas producto de especímenes con DG moderada secretan menor cantidad de insulina por lo que su tolerancia a la glucosa se ve reducida, en casos de diabetes severa en cambio, se produce en las crías una resistencia a la insulina a nivel de la musculatura esquelética y a nivel hepático. Los descendientes que llegan a ser adultos de procreadoras diabéticas muestran una masa de células beta, un nivel de insulina y glucemia normales, pero la respuesta de la insulina a la glucosa se encuentra alterada tanto in vivo como in vitro $[13,28]$

\subsection{Factores genéticos}

Modelos estiman un $38 \%$ en las variaciones observadas en el peso al nacimiento correlacionando con el factor genético materno-fetal. De este 38\%, el 53\% se daría por el genotipo materno, un $39 \%$ por el genotipo fetal y un $5 \%$ por el sexo fetal $[3,7,13,15,28]$. Las variaciones genéticas heredadas explican crecimientos intrauterinos pobres, no correlacionan con la presencia o ausencia de componentes externos [14].

\subsection{Factor nutricional}

La maduración y desarrollo de la placenta se correlaciona con el crecimiento del nuevo ser dentro del útero materno, por lo que cobran mucha importancia los sustratos energéticos como los carbohidratos y lípidos; estructurales como aminoácidos; y otros elementos como agua, oxígeno, oligoelementos entre otros. Se ha estimado que el feto requiere $100 \mathrm{Kcal}$ al día, la conservación del embarazo requiere un aporte energético extra de $136 \mathrm{Kcal} / \mathrm{d}$ ía. Obteniendo $240 \mathrm{Kcal} / \mathrm{d}$ ía en promedio, lo que supone un consumo de 80.000 Kcal durante todo el embarazo [6, 13-15].

\subsection{Factores placentarios}

Diferentes aspectos placentarios contribuyen al crecimiento intrauterino: Como principales se encuentran el aporte de nutrientes y oxígeno, difusión de la circulación materna, eliminación de metabolitos fetales, al actuar como órgano endocrino que repercutirá en el metabolismo materno - fetal. El perfeccionamiento del nuevo ser estará condicionado por los factores placentarios, si existe alguna anomalía como implantación muy temprana o errores en la placentación el desarrollo embrionario y fetal no será el correcto, pudiéndose producir productos bajo peso para la edad gestacional, restricción de crecimiento intrauterino, patologías del embarazo como preeclampsia entre otros $[4-6,9,13,14,23,29]$. 


\subsection{Lactógeno placentario}

El lactógeno placentario (LPh) es una hormona del grupo de los péptidos que se secreta casi exclusivamente en el tejido placentario, al igual que la hormona del crecimiento [23]. El gen que sintetiza el lactógeno placentario es de la familia de la Hormona de Crecimiento, su síntesis es dada en el sincitotrofoblasto desde el quinto y decimo días posterior a la implantación [13, 23].

\subsection{Factores maternos}

Existen al menos tres factores maternos que intervienen con el crecimiento fetal:

- Suministro de nutrientes (estructurales, energéticos) y oxigeno necesarios para el crecimiento fetal y la eliminación de productos de desecho metabólico proveniente del feto [7].

- Presencia de hormonas en la sangre: El LPh y la hormona del Crecimiento $(\mathrm{GH})$ placentaria; aumenta la tasa de secreción de insulina [23].

- En el embarazo las dimensiones del útero aumentan de manera progresiva y de una forma especial en el tercer trimestre, considerado como un factor limitante del crecimiento fetal, como se ha comprobado en embarazos múltiples [3, 12, 22].

\subsection{Aspectos generales de la alimentación en el embarazo}

Lo recomendable es olvidar lo coloquial y no comer por 'dos personas', aunque se puede incrementar levemente el tamaño de las raciones y dividirlo en un número de tomas mayor a lo normal a lo largo del día, aproximadamente cinco o seis veces, con el fin de evitar la ingesta de volumen excesivo de alimentos y reducir subsecuentemente el riesgo de vómitos y pirosis; a nivel hormonal en cambio se ayuda a reducir la demanda de insulina de manera que se evita complicaciones para la madre [13]. La cena es aconsejable que sea ligera con el fin de evitar hipoglucemia inducida por la demanda de sustrato fetal durante el descanso de la madre, asegurando que no se utilice las reservas de proteína y de grasas $[6,13]$.

Los alimentos crudos se deben eliminar de la dieta materna por el riesgo de producirse una intoxicación alimentaria y exposición a infecciones del tipo toxoplasma Gondi; tampoco se deben producir cambios extremos en la alimentación materna o ingerir alimentos que sean extraños o no conocidos para la madre [13].

Generalmente entre los profesionales de la salud que cuidan a las madres en gestación existe un acuerdo para la prescripción de suplementos de hierro, folatos y yodo durante la planificación y el embarazo, considerando las características pregestacionales y gestacionales de cada paciente $[6,7,13,23,28]$.

La Organización Mundial de la Salud (OMS) en 1986 declara que la causa nutricional de retraso mental prevenible más frecuente es la deficiencia del micronutriente yodo, luego de la inanición extrema [28]. Estas recomendaciones describen la importancia del consumo de yodo específicamente, que, desde antes de la gestación, los suplementos 
de yodo se utilizan para incrementar las reservas tiroideas, con el fin de que la población en territorio donde el aporte de yodo sea bajo, se pueda evitar el aparecimiento de enfermedades relacionadas con yodo bajo o el hipotiroidismo congénito [13].

Se aconsejan también la prescripción de suplementos de folatos dado que existe dificultad de mantener niveles adecuados (600 $\mu \mathrm{g} /$ día) mediante la ingesta, además, ayuda a prevenir las malformaciones del tubo neural y anemias macrocíticas e hiperhomocisteinemia por una hipofolatemia. La prescripción de hierro es controvertida puesto que la recuperación de valores de hemoglobina normal y reserva de hierro requiere un tiempo prolongado por lo que pueden aparecer problemas como hemorragias, alteraciones del crecimiento que se observan en fetos con hierro excesivo [7, 13, 28, 30].

No es recomendable el uso de vitaminas sin prescripción establecida o justificada en el periodo de gestación, por el hecho de que el exceso de micronutrientes tiene la capacidad de interferir con la asimilación de uno u otros [13]. El exceso de vitamina A, debido a la teratogenicidad afecta el desarrollo y del crecimiento de la estructura facial; produciendo una disminución del crecimiento y aumento del riesgo de producir espina bífida. Niveles elevados de vitamina B6 se relaciona con alteraciones en el funcionamiento del sistema nervioso. Vitamina C ( $>2 \mathrm{~g} / \mathrm{d}$ ía) en cantidades elevadas se relaciona con escorbuto agudo al nacimiento, puesto que se produce una adaptación fetal a niveles altos de vitamina C [7].

\subsection{El ambiente endocrino uterino clave para un buen desarrollo del páncreas}

El páncreas en desarrollo del feto al término de los primeros tres meses se convierte en un órgano con actividad, que coincide también con la evolución placentaria a la madurez. Por lo mencionado los factores histiotróficos que intervienen en el crecimiento y desarrollo del nuevo ser, son fundamentales en el crecimiento y maduración placentaria y pancreática. Por lo que si existe un ambiente desfavorable y los sustratos necesarios son incapaces de usarse o no están disponibles se producirán anomalías a nivel de transcripción de genes, angiogénesis, producción hormonal, sustratos endocrinos, entre otros $[6,13,23]$. En resumen si en el proceso de desarrollo pancreático existe algún estímulo o agresión, este no se desarrolla de manera adecuada, los estímulos pueden ser exteriores o intrínsecos del feto debido a la programación fetal que se expresan en algún momento del embarazo.

\subsection{Malnutrición o subnutrición materna}

La nutrición se relaciona con niveles de insulina y la sensibilidad de esta, y el número de células beta en los islotes, por lo tanto, si la madre tiene una malnutrición ya sea de índole primaria al mantener déficit de nutrientes en la ingesta o secundaria a circulación materno-fetal deficiente, existe una alteración en el desarrollo pancreático, tanto en su anatomía como en su fisiología con una disminución del número de células especiales. Todos estos cambios se han observado en especímenes roedores, dado que los datos de páncreas fetales en humanos son escasos [13]. 
Un mal desarrollo de la parte endócrina pancreática secundaria a la malnutrición según varios estudios epidemiológicos dará como resultado en el feto una intolerancia a la glucosa en el recién nacido y una diabetes cuando alcance la edad adulta [13]. Esto se da debido a las células beta son altamente sensibles a cambios en la vida intrauterina puesto que son moduladas por factores de transcripción (PDX-1, HNF-3ß) y cuando existen alteraciones en la disponibilidad de sustratos (hipoglucemia, ácidos grasos excesivos) y hormonas aparecen las alteraciones funcionales por la inhibición de los factores mencionados $[9,13]$. Los niveles del factor de crecimiento asociado a la insulina (IGF) y de GH regulan también el desarrollo pancreático, específicamente en el crecimiento de los islotes, por lo tanto, si existe déficit de estas hormonas se produce un círculo vicioso (menos IGF no producen desarrollo de células especializadas, lo que se traduce a menos IGF disponible) $[9,13]$.

\section{Conclusión}

La placenta presenta características genómicas únicas a diferencia del resto de tejidos en el cuerpo humano, gracias a estas peculiaridades se convierte en un tejido sumamente reactivo a las influencias tanto internas como externas, una muy importante es la dieta y nutrición de las mujeres, tanto en el periodo preconcepcional como durante la gestación. Mediante la metilación del ADN se presentan diferentes fenómenos que hacen inhibir o expresar diversos genes contenidos en los tejidos, lo cual tiene gran importancia clínica, especialmente con el crecimiento intrauterino, aparecimiento de defectos del tubo neural durante a vida fetal y el desarrollo de diabetes, hipertensión arterial, obesidad, enfermedades cardiovasculares u otras enfermedades extrauterinas.

\section{Agradecimiento}

Nuestro agradecimiento al Comité Científico organizador del SEMINARIO INTERNACIONAL DE SALUD, ALIMENTACIÓN Y NUTRICIÓN HUMANA SISANH 2020, de la Facultad de Salud Pública de la ESPOCH, por permitir la publicación del presente artículo de revisión y a su Comité Editorial por su gran esfuerzo en pro de la investigación científica y su difusión.

\section{Conflicto de Intereses}

Todos los miembros del grupo de trabajo del presente artículo declaran no tener conflicto alguno de intereses.

\section{Limitación de Responsabilidad}

Todos los enfoques y contenido del presente texto son de completa responsabilidad de los autores. 


\section{Fuentes de Apoyo}

Completamente autofinanciado por los autores.

\section{References}

[1] Pesantez J, García C, Vasquez M, et al. Programación prenatal y epigenética en pequeños rumiantes. Portal Vet. 2019;20-1.

[2] Delahaye F, Do C, Kong Y, et al. Genetic variants influence on the placenta regulatory landscape. PLoS Genet. 2018;14(11):1-34.

[3] Parra B. Nutrición materna y epigenética. Jornada Academica en Epigenetica. 2011.

[4] Casanello P, Castro-Rodríguez JA, Uauy R, Krause BJ. Programación epigenética placentaria en restricción del crecimiento intrauterino. Rev Chil Pediatr. 2016;87(3):154-61.

[5] Carrascosa A. Crecimiento intrauterino: Factores reguladores. Retraso de crecimiento intrauterino. An Pediatr. 2003;58(Suppl. 2):55-73.

[6] Reyes RB, Carrocera LAF. Programación metabólica fetal. Perinatol y Reprod Humana. 2015;29(3):99_ 105.

[7] Oliver Bonet M, Mach N. Factores nutricionales y no nutricionales pueden afectar la fertilidad masculina mediante mecanismos epigenéticos. Nutr Hosp. 2016;33(5):591.

[8] Branco MR, King M, Perez-Garcia V, et al. Maternal DNA Methylation Regulates Early Trophoblast Development. Dev Cell. 2016;36(2):152-63.

[9] Morales E, Vilahur N, Salas LA, et al. Genome-wide DNA methylation study in human placenta identifies novel loci associated with maternal smoking during pregnancy. Int J Epidemiol. 2016;45(5):1644-55.

[10] Rodriguez M, Telles N. La metilación del ADN materno regula el desarrollo temprano del embrión. Rev Investig Clínica. 2004

[11] Krause BJ, Castro-Rodríguez JA, Uauy R, Casanello P. Conceptos generales de epigenética: Proyecciones en pediatriá. Rev Chil Pediatr. 2016;87(1):4-10.

[12] Faingold MC. Factores epigenéticos en mujeres embarazadas con diabetes. Rev Argent Endocrinol Metab. 2014;51(3):151-9.

[13] Sánchez-Muniz FJ, Gesteiro E, Espárrago Rodilla M, Rodríguez Bernal B, Bastida S. La alimentación de la madre durante el embarazo condiciona el desarrollo pancreático, el estatus hormonal del feto y la concentración de biomarcadores al nacimiento de diabetes mellitus y síndrome metabólico. Nutr Hosp. 2013;28(2):250-74.

[14] Robinson WP, Peñaherrera MS, Konwar C, Yuan V, Wilson SL. Epigenetic Modifications in the human placenta. Hum Reprod Prenat Genet. 2019;293-311.

[15] Monk D. Genomic imprinting in the human placenta. Am J Obstet Gynecol. 2015;213(4):S152-62.

[16] Schroeder DI, Lasalle JM. How has the study of the human placenta aided our understanding of partially methylated genes? Epigenomics. 2013;5(6):645-54.

[17] Schroeder DI, Blair JD, Lott P, et al. The human placenta methylome. Proc Natl Acad Sci U SA. 2013;110(15):6037-42.

[18] De Goede OM, Lavoie PM, Robinson WP. Characterizing the hypomethylated DNA methylation profile of nucleated red blood cells from cord blood. Epigenomics. 2016;8(11):1481-94.

[19] Callinan A, Batzer MA. Retrotransposable Elements and Human Disease. Genome. 2006;1:104-15.

[20] Reiss D, Zhang Y, Mager DL. Widely variable endogenous retroviral methylation levels in human placenta. Nucleic Acids Res. 2007;35(14):4743-54.

[21] Nordor A V., Nehar-Belaid D, Richon S, et al. The early pregnancy placenta foreshadows DNA methylation alterations of solid tumors. Epigenetics. 2017;12(9):793-803.

[22] Moleres A, Marti A. Influencia del ambiente y la alimentación en la programación epigenética de la obesidad. Revista Espanola de Obesidad. 2008;6:66-74.

[23] Krause BB, Sobrevia L, Casanello DP. Papel de la placenta en la programaclón fetal de las enfermedades crónlcas del adulto. Impacto del Crecim y Desarro Tempr sobre la salud y bienestar la población. 2009;117-33.

[24] Vasconcelos S, Ramalho C, Marques CJ, Doria S. Altered expression of epigenetic regulators and imprinted genes in human placenta and fetal tissues from second trimester spontaneous pregnancy losses. Epigenetics. 2019;14(12):1234-44.

[25] Koukoura O, Sifakis S, Spandidos DA. DNA methylation in the human placenta and fetal growth (review). Mol Med Rep. 2012;5(4):883-9. 
[26] Rakoczy J, Padmanabhan N, Krzak AM, Kieckbusch J, Cindrova-Davies T, Watson ED. Dynamic expression of TET1, TET2, and TET3 dioxygenases in mouse and human placentas throughout gestation. Placenta. 2017;59:46-56.

[27] Shrestha D, Workalemahu T, Tekola-Ayele F. Maternal dyslipidemia during early pregnancy and epigenetic ageing of the placenta. Epigenetics. 2019;14(10):1030-9.

[28] Carrillo-Gómez CS, Molina-Noyola LD, Torres-Bugarín O. Ácido fólico: Económico modulador de la estabilidad genómica, epigenética y el cáncer; deficiencias, fuentes, efectos adversos por exceso y recomendaciones gubernamentales. El Resid. 2017;12(3):89-103.

[29] Abraham E, Rousseaux S, Agier L, et al. Pregnancy exposure to atmospheric pollution and meteorological conditions and placental DNA methylation. Environ Int. 2018;118:334-47.

[30] Rahat B, Mahajan A, Bagga R, Hamid A, Kaur J. Epigenetic modifications at DMRs of placental genes are subjected to variations in normal gestation, pathological conditions and folate supplementation. Sci Rep. 2017;7(January):1-16 